\title{
ON SYMMETRIC NORM INEQUALITIES AND POSITIVE DEFINITE BLOCK-MATRICES
}

\author{
ANTOINE MHANNA
}

Abstract. The main purpose of this paper is to englobe some new and known types of positive semi definite matrices $M=\left(\begin{array}{cc}A & X \\ X^{*} & B\end{array}\right)$ satisfying or not the inequality $\|M\| \leqslant\|A+B\|$ for all symmetric norms.

Mathematics subject classification (2010): 15A60, 15A42, 47A30.

Keywords and phrases: Symmetric norm, positive semi definite block-matrices, positive partial transpose.

\section{REFERENCES}

[1] F. Zhang, Matrix Theory Basic Results and Techniques, $2^{\text {nd }}$ Edition, (Universitext) Springer, 2011.

[2] J. C. Bourin, E. Y. LEE, AND M. Lin, On a decomposition lemma for positive semi definite blockmatrices, Linear Algebra and its Applications 437, 7 (2012), 1906-1912.

[3] J. C. BOURIN, E. Y. LEE, Unitary orbits of Hermitian operators with convex or concave functions, Bull. London Math. Soc 44, 6 (2012), 1085-1102.

[4] M. Lin, H. Wolkowicz, Hiroshima's theorem and matrix norm inequalities, Acta Sci. Math. (Szeged) 81, 1-2 (2015), 45-53.

[5] M. Lin AND H. WolKowiCZ, An eigenvalue majorization inequality for positive semidefinite block matrices, Linear and Multilinear Algebra 60, 11-12 (2012), 1365-1368. 